\title{
Long noncoding RNA PSMA3-AS1 functions as a microRNA-409-3p sponge to promote the progression of non-small cell lung carcinoma by targeting spindlin 1
}

\author{
LINGLING WANG ${ }^{1}$, LEI WU ${ }^{2}$ and JINFENG PANG ${ }^{3}$ \\ ${ }^{1}$ Precision Medical Center, Affiliated Hospital of Beihua University, Jilin, Jilin 132011; \\ ${ }^{2}$ Department of Thoracic Surgery, Jilin City Central Hospital, Capital Medical University, Jilin, Jilin 132010; \\ ${ }^{3}$ Department of Neurosurgery, Affiliated Hospital of Beihua University, Jilin, Jilin 132011, P.R. China
}

Received March 4, 2020; Accepted June 9, 2020

DOI: $10.3892 / o r .2020 .7693$

\begin{abstract}
PSMA3 antisense RNA 1 (PSMA3-AS1), a long noncoding RNA, promotes the progression of esophageal squamous cell carcinoma. However, no study to date has explored the expression or roles of PSMA3-AS1 in non-small cell lung carcinoma (NSCLC). The present study examined the expression profile and role of PSMA3-AS1 in NSCLC. It also aimed to identify how PSMA3-AS1 promotes the malignant phenotype of NSCLC cells. PSMA3-AS1 expression in NSCLC tissues and cell lines was measured by reverse transcription-quantitative polymerase chain reaction. Cell Counting Kit-8, cell apoptosis, Transwell migration and invasion, and xenograft tumor assays were conducted to study the effects of PSMA3-AS1 on the aggressive phenotype of NSCLC cells. Furthermore, bioinformatics analysis, RNA immunoprecipitation, luciferase reporter assay, western blotting, and rescue experiments were used to elucidate the interaction among PSMA3-AS1, microRNA-409-3p (miR-409-3p), and spindlin 1 (SPIN1) in NSCLC cells. In the present study, high levels of PSMA3-AS1 were confirmed in both NSCLC tissues and cell lines. An increased PSMA3-AS1 level was correlated with advanced tumor-node-metastasis stage and increased lymph node metastasis. Patients with NSCLC with high PSMA3-AS1 levels had shorter overall survival than those with low PSMA3-AS1 levels. PSMA3-AS1 depletion significantly decreased NSCLC cell proliferation, migration, and invasion, as well as substantially increased cell apoptosis in vitro. Furthermore, PSMA3-AS1 deficiency decreased NSCLC tumor growth in vivo. Through molecular mechanism assays, it was revealed that PSMA3-AS1 acted as a molecular
\end{abstract}

Correspondence to: Dr Jinfeng Pang, Department of Neurosurgery, Affiliated Hospital of Beihua University, 12 Jiefang Middle Road, Jilin, Jilin 132011, P.R. China

E-mail: pangjf_surgery@163.com

Key words: PSMA3 antisense RNA 1, lung cancer, long noncoding RNAs sponge for miR-409-3p and consequently increased SPIN1 expression. Notably, rescue experiments revealed that the inhibition of miR-409-3p or restoration of SPIN1 expression abrogated the effects of PSMA3-AS1 knockdown in NSCLC cells. Collectively, PSMA3-AS1 functioned as an oncogenic long noncoding RNA in NSCLC. PSMA3-AS1 sponged miR-409-3p and thus increased SPIN1 expression, promoting the aggressive phenotype of NSCLC cells.

\section{Introduction}

Lung cancer is one of the most frequent human malignancies with the highest global incidence rate and mortality (1). Approximately 246,220 newly diagnosed cases and 147,510 mortalities due to respiratory system cancer are estimated to occur worldwide each year (2). Clinically, non-small cell lung carcinoma (NSCLC) is the major pathology subtype of lung cancer, accounting for approximately $75-80 \%$ of all lung cancer cases (3). Approximately $75 \%$ of patients with NSCLC are diagnosed at an advanced stage, which indicates that most patients are not completely cured after conventional treatments (4). Despite the integrated use of surgical resection, radiochemotherapy, and targeted therapies, the outcomes of patients with NSCLC remain unsatisfactory (5). The overall 5 -year survival rate of patients with NSCLC is approximately $15 \%$, which has not noticeably improved in the past few decades (6). In view of this, there is a great demand to better understand NSCLC pathogenesis in order to identify promising diagnostic methods and therapeutic techniques.

Long noncoding RNAs (lncRNAs) are a group of noncoding RNA transcripts longer than 200 nucleotides that are not encoded into proteins (7). They are identified as important contributors in modulating gene expression via chromatin modification, transcriptional, post-transcriptional, and translational regulation (8). IncRNAs have gained increasing attention due to their roles in carcinogenesis and cancer progression (9). Several lncRNAs are aberrantly expressed in NSCLC. MIR503HG (10), LINC00261 (11), and TOB1-AS1 (12) are downregulated in NSCLC, whereas SNHG16 (13), FTH1P3 (14), and KCNQ1OT1 (15) are highly expressed. The pro-oncogenic and anti-oncogenic activities of 
lncRNAs are known to be implicated in NSCLC genesis and progression $(16,17)$.

MicroRNAs (miRNAs) are an abundant family of highly conserved, noncoding, and single-stranded RNA molecules with lengths ranging from 18 to 25 nucleotides (18). miRNAs post-transcriptionally regulate gene expression by complementarily binding to the 3'-untranslated region (3'-UTR) of their target mRNAs, which consequently results in degradation and/or translation suppression (19). An increasing amount of evidence highlights that lncRNAs may be capable of directly interacting with miRNAs by functioning as competitive endogenous RNAs (ceRNAs) or molecular sponges to regulate their target genes (20-22). Therefore, an in-depth exploration of tumor-related lncRNAs and miRNAs in NSCLC, as well as of the mechanisms underlying their roles, may be of great significance in cancer diagnosis, therapy, and prevention.

PSMA3 antisense RNA 1 (PSMA3-AS1), an IncRNA, has been revealed to promote the progression of esophageal squamous cell carcinoma (23). However, there are no studies to date that explore the expression or roles of PSMA3-AS1 in NSCLC. In the present study, the expression profile and roles of PSMA3-AS1 in NSCLC were determined. It was also revealed how PSMA3-AS1 regulates the malignant phenotype of NSCLC cells.

\section{Materials and methods}

Sample collection. NSCLC tissues and adjacent normal tissues were collected from 61 patients (age range, 52-77 years; 32 male and 29 female patients) with NSCLC who were admitted to the Affiliated Hospital of Beihua University between June 2014 and February 2015. All participants were diagnosed with primary NSCLC and had not been treated with preoperative radiotherapy, chemotherapy, immunotherapy, or targeted therapy. All tissue samples were immediately frozen in liquid nitrogen after surgical excision and stored in liquid nitrogen until use. All participants provided written informed consent, and the present study was approved by the Ethics Committee of the Affiliated Hospital of Beihua University. All experimental steps were conducted in accordance with the Declaration of Helsinki.

Cell lines and culture conditions. The human non-tumorigenic bronchial epithelium cell line BEAS-2B was acquired from American Type Culture Collection, and NSCLC cell lines (A549, SK-MES-1, H1703, H460 and H522) were obtained from Shanghai Academy of Life Sciences, the Chinese Academy of Sciences. BEAS-2B cells were grown in BEGM ${ }^{\mathrm{TM}}$ Bronchial Epithelial Cell Growth Medium (Lonza Group, Ltd.; Clonetics Corporation), while SK-MES-1 cells were cultured in Minimum Essential Medium (Gibco; Thermo Fisher Scientific, Inc.). The other four NSCLC cell lines were cultured in Roswell Park Memorial Institute (RPMI)-1640 media (Gibco; Thermo Fisher Scientific, Inc.). All culture media was supplemented with $10 \%$ fetal bovine serum (FBS; Gibco; Thermo Fisher Scientific, Inc.) and a $1 \%$ penicillin/streptomycin mixed solution (TransGen Biotech Co., Ltd.). All cells were incubated in humidified air with $5 \% \mathrm{CO}_{2}$ at $37^{\circ} \mathrm{C}$.

Cell transfection. Small interfering RNAs (siRNAs) targeting PSMA3-AS1 (si-PSMA3-AS1\#1, si-PSMA3-AS1\#2, and
si-PSMA3-AS1\#3) and negative control (si-NC) were synthesized by Shanghai GenePharma Co., Ltd.. The si-PSMA3-AS1\#1 sequence was 5'-CTGATTTTATGGGAA AATTAAGC-3'; the si-PSMA3-AS1\#2 sequence was 5'-AAC TTGAAAAGCACATTTTAAAT-3'; the si-PSMA3-AS1\#3 sequence was 5'-TAGTTAAGTTCTGTATTTAGTGA-3'; and the si-NC sequence was 5'-CACGATAAGACAATG TATTT-3'. miR-493-3p mimic (cat. no. miR10003161-1-5), scrambled mimic control (cat. no. miR1N0000001-1-5; miR-NC), miR-493-3p inhibitor (cat. no. miR20003161-1-5), and negative control inhibitor (cat. no. miR2N0000001-1-5; $\mathrm{NC}$ inhibitor) were acquired from Guangzhou RiboBio Co., Ltd. The spindlin 1 (SPIN1) overexpression plasmid (pcDNA3.1/SPIN1) and empty pcDNA3.1 plasmid were designed and generated by Shanghai GenePharma Co., Ltd.. Cells were inoculated into 6-well plates with a density of $8 \times 10^{5}$ cells/well, and transfected with miRNA mimic $(50 \mathrm{nM})$, miRNA inhibitor (50 nM), siRNA (100 nM), or plasmid $(4 \mu \mathrm{g})$ using the Lipofectamine $2000^{\mathrm{TM}}$ reagent (Invitrogen; Thermo Fisher Scientific, Inc.). Cell Counting Kit-8 (CCK-8) and cell apoptosis assays were conducted after 24 and $48 \mathrm{~h}$ of incubation, respectively. Transwell migration and invasion assays and reverse transcription-quantitative polymerase chain reaction (RT-qPCR) were performed at $48 \mathrm{~h}$ post-transfection. Seventy-two hours later, western blotting was carried out to detect protein expression.

Subcellular fractionation. A Cytoplasmic and Nuclear RNA Purification Kit (Norgen Biotek Corp.) was used to isolate nuclear and cytosolic fractions in NSCLC cells. The relative expression of PSMA3-AS1 in nuclear and cytosolic fractions was analyzed by RT-qPCR. U6 small nuclear RNA and glyceraldehyde-3-phosphate dehydrogenase (GAPDH) were used as the nuclear and cytoplasmic controls, respectively.

$R T$ - $q P C R$. The extraction of total RNA was conducted using TRIzol (Invitrogen; Thermo Fisher Scientific, Inc.). To quantify PSMA3-AS1 and SPIN1, complementary DNA was synthesized by reverse transcription using a PrimeScript RT Reagent Kit (Takara Bio.). The temperature protocol for reverse transcription was as follows: $37^{\circ} \mathrm{C}$ for $15 \mathrm{~min}$ and $85^{\circ} \mathrm{C}$ for $5 \mathrm{sec}$. The complementary DNA was then used as a template to determine the expression of target genes using SYBR Premix Ex Taq (Takara Bio). The thermocycling conditions for quantitative PCR were as follows: $5 \mathrm{~min}$ at $95^{\circ} \mathrm{C}$, followed by 40 cycles at $95^{\circ} \mathrm{C}$ for $30 \mathrm{sec}$ and $65^{\circ} \mathrm{C}$ for $45 \mathrm{sec}$, and $50^{\circ} \mathrm{C}$ for $30 \mathrm{sec}$. The expression of PSMA3-AS1 and SPIN1 was normalized to that of GAPDH. To determine miR-409-3p expression, total RNA was reverse transcribed into complementary DNA using a miScript Reverse Transcription Kit (Qiagen GmbH). The temperature protocols for reverse transcription were as follows: $37^{\circ} \mathrm{C}$ for $60 \mathrm{~min}, 95^{\circ} \mathrm{C}$ for $5 \mathrm{~min}$ and maintenance at $4^{\circ} \mathrm{C}$. Quantitative PCR was conducted using a miScript SYBR Green PCR Kit (Qiagen GmbH). The thermocycling conditions for quantitative PCR were as follows: $95^{\circ} \mathrm{C}$ for $2 \mathrm{~min}, 95^{\circ} \mathrm{C}$ for $10 \mathrm{sec}, 55^{\circ} \mathrm{C}$ for $30 \mathrm{sec}$ and $72^{\circ} \mathrm{C}$ for $30 \mathrm{sec}$, for 40 cycles. U6 was used as the control for miR-409-3p expression. The $2^{-\Delta \Delta C q}$ method (24) was applied to calculate relative gene expression.

The primers were designed as follows: PSMA3-AS1, 5'-TTC CTCCAGGACAGCACCTAGT-3' (forward) and 5'-CGTCTC 
TGATGTGGCTTATACGA-3' (reverse); SPIN1, 5'-CCAACA TGATGAAGAAGAGGACAT-3' (forward) and 5'-AGGATT TACAGGCACCTGGTCC-3' (reverse); miR-409-3p, 5'-TCG GCAGGAGGUUACCCGAGCA-3' (forward) and 5'-CAC TCAACTGGTGTCGTGGA-3' (reverse); U6, 5'-GCTTCG GCAGCACATATACTAAAAT-3' (forward) and 5'-CGC TTCACGAATTTGCGTGTCAT-3' (reverse); and GAPDH, 5'-CGGAGTCAACGGATTTGGTCGTAT-3' (forward) and 5'-AGCCTTCTCCATGGTGGTGAAGAC-3' (reverse).

CCK-8 assay. The proliferative ability of NSCLC cells was evaluated using CCK-8 (Dojindo Molecular Technologies, Inc.). Transfected cells were collected after $24 \mathrm{~h}$ of culture and seeded into a 96 -well plate at a density of $2 \times 10^{3}$ cells/well. To assess cell proliferation, $10 \mu \mathrm{l}$ of the CCK-8 solution was added into each well, and the plates were incubated in humidified air with $5 \% \mathrm{CO}_{2}$ at $37^{\circ} \mathrm{C}$. The optical density at a wavelength of $450 \mathrm{~nm}$ in each well was measured using a microplate reader (Bio-Rad Laboratories, Inc.). Cellular proliferation was detected at four time points $(0,1,2$ and 3 days after cell inoculation), and the growth curves were plotted according to the measurement data.

Cell apoptosis assay. After $48 \mathrm{~h}$ of culture, transfected cells $\left(\sim 1.5 \times 10^{6}\right.$ cells $)$ were collected and rinsed with ice-cold phosphate buffer solution (Gibco, Thermo Fisher Scientific, Inc). Following centrifugation at $4^{\circ} \mathrm{C}$, cells were resuspended in $100 \mu \mathrm{l}$ of binding buffer from the Annexin V-Fluorescein Isothiocyanate (FITC) Apoptosis Detection Kit (Biolegend) and double-stained with $5 \mu 1$ of FITC-Annexin V and $5 \mu \mathrm{l}$ of propidium iodide at room temperature in the dark. The apoptosis rate was examined using a flow cytometer (FACScan; BD Biosciences). Cell Quest software (version 5.1; BD Biosciences) was used for data analysis.

Transwell migration and invasion assays. The migratory ability of NSCLC cells was determined using Transwell chambers ( $8 \mu \mathrm{m}$; BD Biosciences). The Transwell invasion assay was performed with experimental steps that were similar to the migration assay, except that Matrigel (BD Biosciences) was used to coat the chambers. In brief, $100 \mu \mathrm{l}$ of FBS-free culture medium containing $5 \times 10^{4}$ cells were added into the upper compartments, while the lower compartments were covered with culture medium supplemented with $20 \%$ FBS, which was used as a chemoattractant. Following a 24-h incubation, the non-migrated and non-invaded cells were gently removed with a cotton swab, whereas the migrated and invaded cells were fixed with $4 \%$ paraformaldehyde at room temperature for $20 \mathrm{~min}$ and stained with $0.1 \%$ crystal violet at room temperature for $20 \mathrm{~min}$. An inverted light microscope (x200 magnification; Olympus Corporation) was used to image and count the number of migrated and invaded cells.

Xenograft tumor assay. A short hairpin RNA (shRNA) targeting PSMA3-AS1 (sh-PSMA3-AS1) and negative control (sh-NC) were synthesized by Shanghai GenePharma Co., Ltd. and used for lentivirus production. $\mathrm{H} 460$ cells were transfected with lentivirus expressing sh-PSMA3-AS1 or sh-NC, and the stably transfected cells were selected using puromycin. The sh-PSMA3-AS1 sequence was 5'-CCGGCTAATTTTATG
GGAAAATTAAGCCTCGAGGCTTAATTTTCCCATAA AATCAGTTTTTG-3' and the sh-NC sequence was 5'-CCG GCACGATAAGACAATGTATTTCTCGAGAAATACATT GTCTTATCGTGTTTTTG-3'.

Six-week-old male BALB/c nude mice (total number, 6; weight, $20 \mathrm{~g}$ ) were purchased from SLAC Laboratory Animal Co., Ltd., and kept under pathogen-free conditions at $25^{\circ} \mathrm{C}$ with $50 \%$ humidity, with a 10/14-h light/dark cycle and ad libitum food/water access. Animal experiments were performed under the approval of the Animal Ethics Committee of the Affiliated Hospital of Beihua University. H460 cells that stably expressed sh-PSMA3-AS1 or sh-NC were subcutaneously injected into the flank of nude mice. After 8 days, tumor size was recorded every 5 days and used for calculating the tumor volume with a simplified equation (tumor volume $=$ length $\mathrm{x}$ width ${ }^{2} \mathrm{x} 0.5$ ). All mice were euthanized by cervical dislocation at 4 weeks post-injection, and tumor xenografts were excised and weighed.

Bioinformatics analysis. StarBase version 3.0 (http://starbase. sysu.edu.cn/) was used to predict the target miRNA(s) of PSMA3-AS1.

RNA immunoprecipitation (RIP). The Magna-RIP RNA Binding Immunoprecipitation Kit (EMD Millipore) was used to verify the interaction between PSMA3-AS1 and miR-409-3p. NSCLC cells were collected and lysed in radioimmunoprecipitation assay (RIPA) buffer that was supplemented with a protease inhibitor cocktail and RNase inhibitor. Cell lysates were collected and incubated with magnetic beads coated with Anti-Argonaute2 (Anti-Ago2) or IgG (both from EMD Millipore) at $4^{\circ} \mathrm{C}$ overnight. The coprecipitated RNA was extracted and used in RT-qPCR to determine the expression of PSMA3-AS1 and miR-409-3p.

Luciferase reporter assay. The sequences of wild-type (WT) PSMA3-AS1 containing miR-409-3p binding sequences and mutant (MUT) PSMA3-AS1 fragments were synthesized by Shanghai GenePharma Co., Ltd. and inserted into a pmirGLO luciferase reporter vector (Promega Corporation), generating WT-PSMA3-AS1 and MUT-PSMA3-AS1. NSCLC cells were seeded into 24 -well plates with a density of $1.5 \times 10^{5}$ cells/well. After overnight culture at $37^{\circ} \mathrm{C}$, cells were co-transfected with WT-PSMA3-AS1 $(1.6 \mu \mathrm{g})$ or MUT-PSMA3-AS1 $(1.6 \mu \mathrm{g})$ and miR-409-3p mimic $(50 \mathrm{nM})$ or miR-NC $(50 \mathrm{nM})$ using Lipofectamine ${ }^{\mathrm{TM}}$ 2000. Following $48 \mathrm{~h}$ of culture, a Dual-Luciferase Reporter assay system (Promega Corporation) was used to assess luciferase activity. Firefly luciferase activity was normalized to Renilla luciferase activity.

Western blotting. Total protein was isolated from cultured cells using RIPA buffer (Beyotime Institute of Biotechnology). After quantification by the bicinchoninic acid assay (Beyotime Institute of Biotechnology), equal amounts of protein $(30 \mu \mathrm{g})$ were separated by $10 \%$ sodium dodecyl sulfate-polyacrylamide gel electrophoresis and subsequently transferred onto polyvinylidene difluoride membranes. The membranes were then blocked with 5\% non-fat milk powder at room temperature for $2 \mathrm{~h}$ and incubated with primary antibodies overnight at $4^{\circ} \mathrm{C}$. Rabbit anti-human SPIN1 (product code ab118784) and rabbit anti-human GAPDH (product code ab181603; both 
A

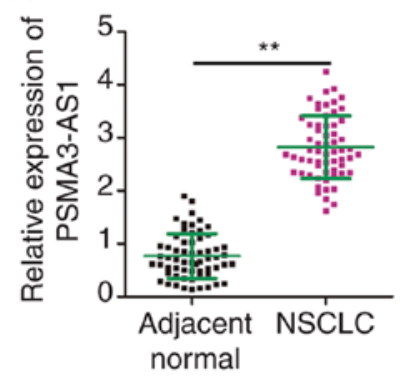

B

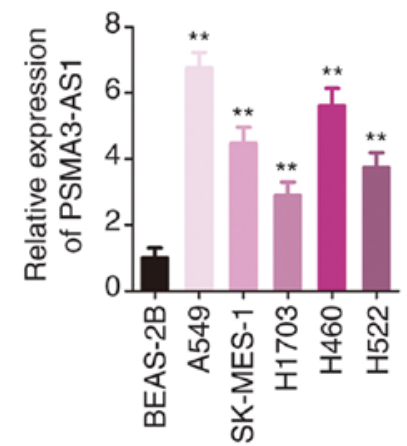

C

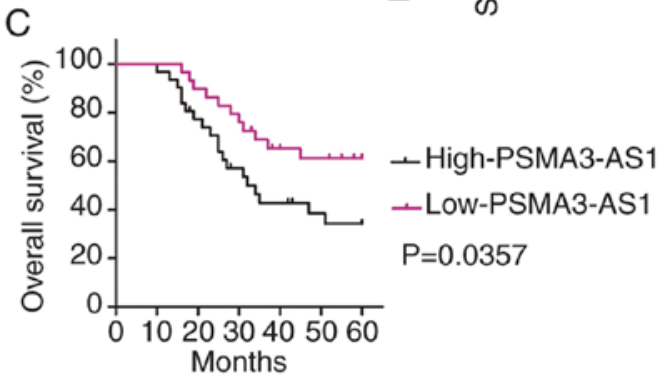

Figure 1. PSMA3-AS1 is upregulated in NSCLC and negatively related to clinical outcomes of patients. (A) A total of 61 pairs of NSCLC tissues and adjacent normal tissues were collected and used to detect PSMA3-AS1 expression via RT-qPCR. (B) The expression of PSMA3-AS1 was detected in five NSCLC cell lines (A549, SK-MES-1, H1703, H460, and H522) by RT-qPCR. A human non-tumorigenic bronchial epithelium cell line BEAS-2B was used as the control. (C) The survival rate of high-expression and low-expression PSMA3-AS1 groups was examined by the Kaplan-Meie method and log-rank test $(\mathrm{P}=0.0357){ }^{* * *} \mathrm{P}<0.01$. PSMA3-AS1, PSMA3 antisense RNA 1; NSCLC, non-small cell lung carcinoma; RT-qPCR, reverse transcription-quantitative polymerase chain reaction.

from Abcam) antibodies were used with a dilution of 1:1,000. Membranes were then incubated with a goat anti-rabbit horseradish peroxidase-conjugated antibody (product code ab205718; 1:5,000 dilution; Abcam), after which the protein signals were detected using an Enhanced Chemiluminescence Western Blotting Detection Reagent (GE Healthcare Life Sciences). Quantity One software (version 4.62; Bio-Rad Laboratories, Inc.) was utilized for densitometric analysis.

Statistical analysis. All data are presented as the means \pm standard deviation and analyzed with SPSS version 13.0 software (SPSS, Inc.). Pearson's correlation coefficient analysis was performed to examine the correlation between PSMA3-AS1 and miR-409-3p in NSCLC tissues. The $\chi^{2}$ test was used to test the association between PSMA3-AS1 expression and clinicopathological factors in patients with NSCLC. Student's t-test was performed to analyze the differences between two groups, whereas one-way analysis of variance with Tukey's post hoc test was used for comparing the data among multiple groups. Survival curves were plotted using the Kaplan-Meier method, after which the survival curves were compared with the log-rank test. A P-value $<0.05(\mathrm{P}<0.05)$ was considered to indicate a statistically significant difference.

\section{Results}

PSMA3-AS1 is upregulated in NSCLC and negatively associated to the survival of patients. First, RT-qPCR was
Table I. Association between PSMA3-AS1 expression and clinicopathological factors in NSCLC.

\begin{tabular}{lccc}
\hline & \multicolumn{2}{c}{ PSMA3-AS1 } & \\
\cline { 2 - 3 } & $\begin{array}{c}\text { High } \\
\text { Factors }\end{array}$ & $\begin{array}{c}\text { Low } \\
(\mathrm{n}=30)\end{array}$ & P-value \\
\hline Sex & & & 0.164 \\
$\quad$ Male & 17 & 15 & \\
Female & 14 & 15 & \\
Age (years) & & & 0.779 \\
$<55$ & 11 & 13 & \\
$\geq 55$ & 20 & 17 & \\
Smoking history & & & 0.267 \\
Smokers & 14 & 12 & \\
Non-smokers & 17 & 18 & \\
Tumor size (cm) & & & 0.073 \\
$<3$ & 10 & 17 & \\
$\geq 3$ & 21 & 13 & \\
TNM stage & & & 0.040 \\
I-II & 13 & 21 & \\
III-IV & 18 & 9 & \\
Lymph node metastasis & & & 0.018 \\
$\quad$ Negative & 14 & 23 & \\
Positive & 17 & 7 & \\
\hline
\end{tabular}

PSMA3-AS1, PSMA3 antisense RNA 1; NSCLC, non-small cell lung carcinoma; TNM, tumor-node-metastasis.

performed to determine PSMA3-AS1 expression in 61 pairs of NSCLC tissues and adjacent normal tissues. The data confirmed the higher expression of PSMA3-AS1 in NSCLC tissues relative to that in adjacent normal tissues (Fig. 1A). In addition, PSMA3-AS1 expression in a panel of NSCLC cell lines (A549, SK-MES-1, H1703, H460 and H522) and a human non-tumorigenic bronchial epithelium cell line BEAS-2B was investigated via RT-qPCR. PSMA3-AS1 expression was significantly increased in the five examined NSCLC cell lines compared with in BEAS-2B cells (Fig. 1B).

The median value of PSMA3-AS1 expression in NSCLC tissues was regarded as the cut-off line, and all enrolled patients with NSCLC were accordingly classified into either low-PSMA3-AS1 $(n=30)$ or high-PSMA3-AS1 $(n=31)$ groups. Increased PSMA3-AS1 expression was significantly associated with tumor-node-metastasis (TNM) stage $(\mathrm{P}=0.040)$, and lymph node metastasis $(\mathrm{P}=0.018)$ in the 61 patients with NSCLC (Table I). Furthermore, patients with NSCLC with high PSMA3-AS1 expression had a relatively shorter overall survival than the patients with low PSMA3-AS1 expression (Fig. 1C; $\mathrm{P}=0.0357$ ). These results indicated that PSMA3-AS1 was upregulated in NSCLC and negatively associated with the clinical outcomes of patients.

PSMA3-AS1 depletion inhibits proliferation, migration, and invasion and increases apoptosis in NSCLC cells. Since A549 and $\mathrm{H} 460$ cell lines exhibited the most significant upregulation of 

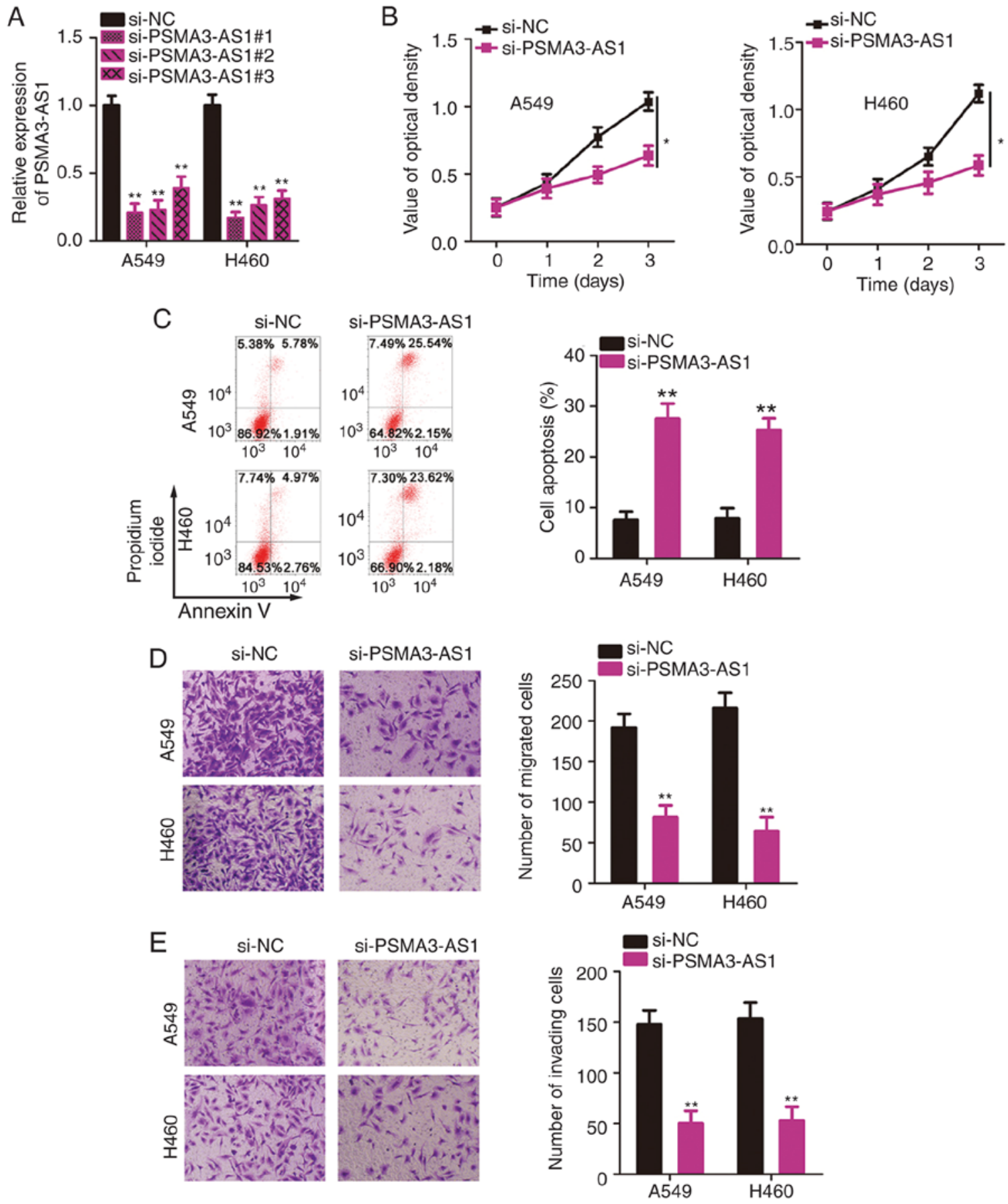

Figure 2. Downregulation of PSMA3-AS1 inhibits the aggressive phenotype of NSCLC cells. (A) RT-qPCR was used to quantify PSMA3-AS1 in A549 and H460 cells after si-PSMA3-AS1 or si-NC transfection. (B) Cell proliferation was detected via the CCK-8 assay in A549 and H460 cells transfected with si-PSMA3-AS1 or si-NC. (C) The apoptosis of PSMA3-AS1-deficient A549 and H460 cells was assessed by flow cytometric analysis. (D and E) The migration and invasion of si-PSMA3-AS1-transfected or si-NC-transfected A549 and $\mathrm{H} 460$ cells were evaluated by Transwell migration and invasion assays. "P<0.05 and ${ }^{* *} \mathrm{P}<0.01$. PSMA3-AS1, PSMA3 antisense RNA 1; NSCLC, non-small cell lung carcinoma; RT-qPCR, reverse transcription-quantitative polymerase chain reaction; CCK-8, Cell Counting Kit-8.

PSMA3-AS1 expression among the five NSCLC cell lines, these two cell lines were selected for subsequent functional assays. To investigate the precise roles of PSMA3-AS1 in NSCLC, three siRNAs targeting PSMA3-AS1 (si-PSMA3-AS1\#1, si-PSMA3-AS1\#2, and si-PSMA3-AS1\#3) were designed and transfected into A549 and $\mathrm{H} 460$ cells. The transfection efficiencies were evaluated by RT-qPCR analysis. A substantial decrease in PSMA3-AS1 was confirmed in A549 and H460 cells after transfection with si-PSMA3-AS1 (Fig. 2A). si-PSMA3-AS1\#1 was the most effective at silencing PSMA3-AS1 expression and therefore was renamed si-PSMA3-AS1 and used in the loss-of-function experiments.

The CCK-8 assay was conducted to evaluate the proliferation of A549 and H460 cells after PSMA3-AS1 knockdown. Transfection with si-PSMA3-AS1 significantly inhibited the proliferative ability of A549 and H460 cells relative to that in si-NC-transfected cells (Fig. 2B). In addition, knockdown of PSMA3-AS1 resulted in increased apoptosis of A549 

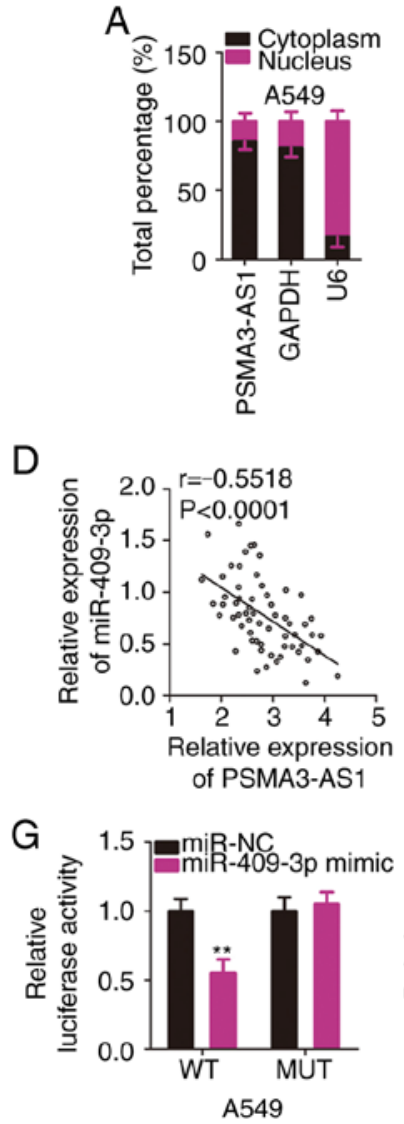

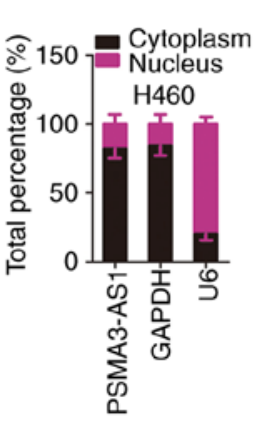

E
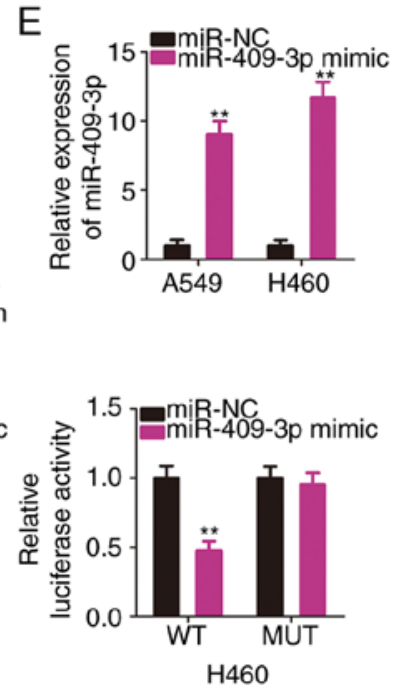

B

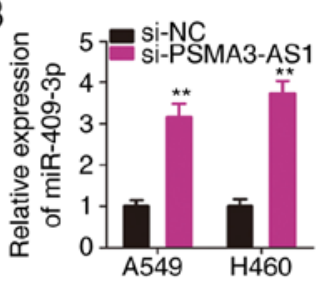

C

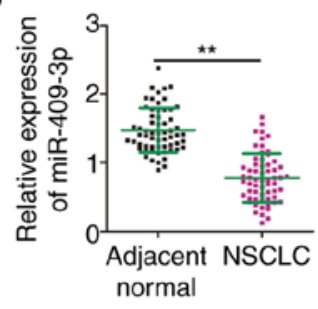

$\mathrm{F}$

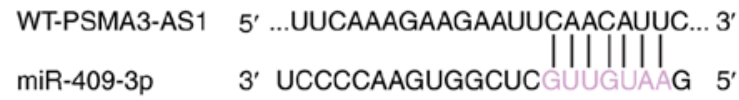

MUT-PSMA3-AS1 5' ...UUCAAAGAAGAAUU GUUGUAAC... 3'
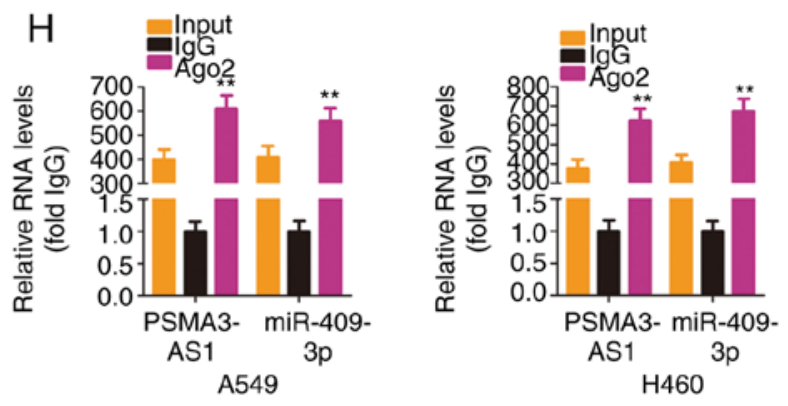

Figure 3. PSMA3-AS1 acts as a sponge for miR-409-3p in NSCLC cells. (A) Subcellular fractionation was used to identify the localization of PSMA3-AS1 in NSCLC cells. (B) Expression of miR-409-3p in A549 and H460 cells after si-PSMA3-AS1 or si-NC transfection was measured by RT-qPCR. (C) RT-qPCR was used to determine miR-409-3p expression in 61 pairs of NSCLC tissues and adjacent normal tissues. (D) The relationship between PSMA3-AS1 and miR-409-3p in the 61 NSCLC tissues was confirmed by Pearson's correlation coefficient analysis $(r=-0.5518, P<0.0001)$. (E) The transfection efficiency of miR-409-3p mimic in A549 and H460 cells was assessed by RT-qPCR analysis. (F) The predicted binding site for miR-409-3p within PSMA3-AS1. The mutant binding sequences are also presented. (G) A549 and H460 cells were transfected with WT-PSMA3-AS1 or MUT-PSMA3-AS1 in the presence of miR-409-3p mimic or miR-NC. A luciferase assay was used to assess the binding interaction between miR-409-3p and PSMA3-AS1. (H) The RIP assay was conducted in A549 and H460 cells, and the co-precipitated RNA was used to quantify miR-409-3p and PSMA3-AS1 overexpression using RT-qPCR. ** $<0.01$. PSMA3-AS1, PSMA3 antisense RNA 1; miR-409-3p, microRNA-409-3p; NSCLC, non-small cell lung carcinoma; RT-qPCR, reverse transcription-quantitative polymerase chain reaction; WT, wild-type; RIP, RNA immunoprecipitation.

and H460 cells compared with the si-NC group (Fig. 2C), as evidenced by flow cytometric analysis. Furthermore, the migration and invasion abilities of NSCLC cells were analyzed by Transwell assays, which revealed that knockdown of PSMA3-AS1 caused a significant decrease in the migratory (Fig. 2D) and invasive (Fig. 2E) abilities of A549 and H460 cells. To summarize, PSMA3-AS1 downregulation inhibited the aggressive phenotype of NSCLC cells.

PSMA3-AS1 acts as a molecular sponge for miR-409-3p and negatively regulates its levels in NSCLC. To reveal the molecular mechanism of PSMA3-AS1 in NSCLC, subcellular fractionation was used to analyze the expression distribution of PSMA3-AS1 in A549 and H460 cells. PSMA3-AS1 was mostly distributed in the cytoplasm of A549 and H460 cells (Fig. 3A). Mechanistically, it is widely accepted that cytoplasmic lncRNAs are implicated in the regulation of target genes by sponging miRNAs (25). Accordingly, it was presumed that PSMA3-AS1 may function as a ceRNA or molecular sponge for specific miRNAs in NSCLC cells.

To test the aforementioned hypothesis, bioinformatics analysis was performed to identify putative miRNAs harboring binding site(s) in PSMA3-AS1. Table SI indicates all the putative miRNAs harboring binding sites within PSMA3-AS1. miR-409-3p is known to exhibit cancer-inhibiting roles in NSCLC (26), and therefore, it was selected for subsequent experiments. The RT-qPCR results revealed that miR-409-3p expression was significantly increased in A549 and H460 cells upon PSMA3-AS1 knockdown (Fig. 3B). In addition, miR-409-3p was significantly downregulated in NSCLC tissues compared with in adjacent normal tissues (Fig. 3C). Furthermore, an inverse correlation between the levels of miR-409-3p and PSMA3-AS1 in the 61 NSCLC tissues was demonstrated by Pearson's correlation coefficient analysis (Fig. 3D; $r=-0.5518, \mathrm{P}<0.0001$ ).

To conduct follow-up assays, miR-409-3p mimic was used to overexpress miR-409-3p in A549 and H460 cells, which was confirmed by RT-qPCR analysis (Fig. 3E). A luciferase reporter assay was then conducted to confirm the binding interaction between miR-409-3p and PSMA3-AS1 in NSCLC cells. The WT and MUT binding sequences between miR-409-3p and PSMA3-AS1 are presented in Fig. 3F. The results of the luciferase reporter assays demonstrated that exogenous miR-409-3p expression suppressed the luciferase 
A

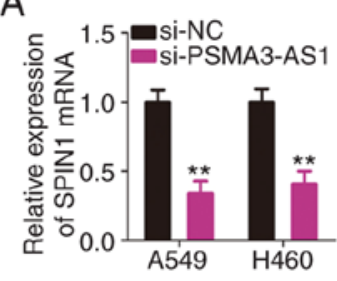

$\mathrm{B}$

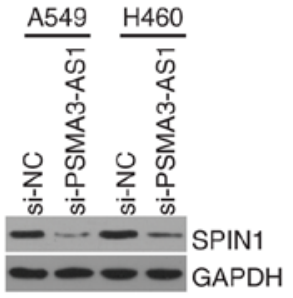

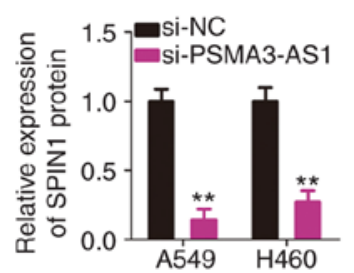

E $\quad$ NC inhibitor

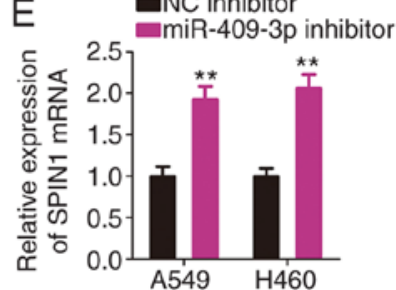

F

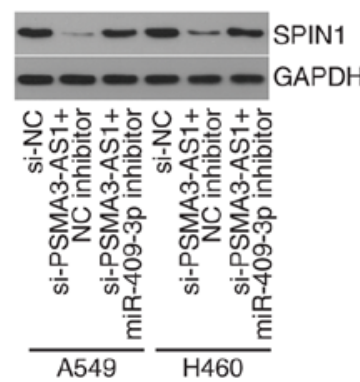

D
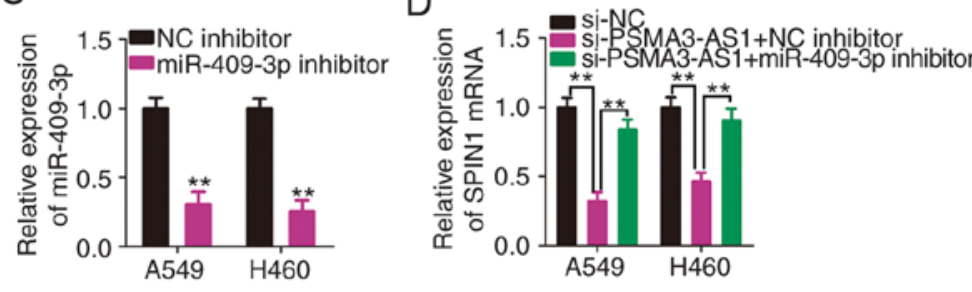

Si-NC

si-PSMA3-AS1+NC inhibitor si-PSMA3-AS1+miR-409-3p inhibitor

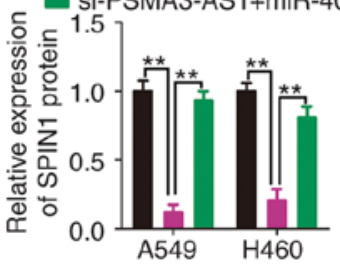

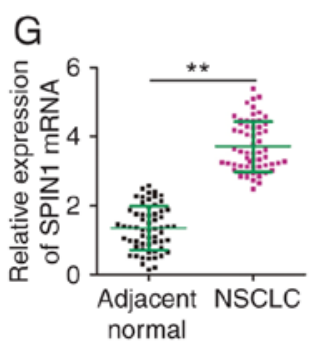

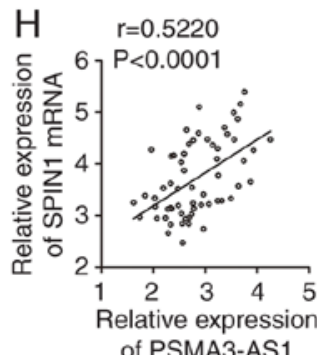

Figure 4. PSMA3-AS1 sponges miR-409-3p and consequently increases SPIN1 expression in NSCLC cells. (A and B) RT-qPCR and western blotting were used to determine SPIN1 mRNA and protein expression in A549 and H460 cells that were treated with si-PSMA3-AS1 or si-NC. (C) RT-qPCR of the miR-409-3p level in A549 and H460 cells after miR-409-3p inhibitor or NC inhibitor transfection. (D) The miR-409-3p inhibitor or NC inhibitor was transfected into PSMA3-AS1-depleted A549 and H460 cells. The expression level of SPIN1 mRNA was measured by RT-qPCR. (E) A549 and H460 cells were transfected with miR-409-3p inhibitor or NC inhibitor, and RT-qPCR was conducted to determine SPIN1 mRNA expression. (F) Western blotting determined the protein level of SPIN1 in A549 and H460 cells after co-transfection of miR-409-3p inhibitor or NC inhibitor and si-PSMA3-AS1. (G) Total RNA was extracted from 61 pairs of NSCLC tissues and adjacent normal tissues and used for the detection of SPIN1 mRNA via RT-qPCR. (H) Pearson's correlation coefficient analysis revealed the relationship between PSMA3-AS1 and SPIN1 mRNA expression in the 61 NSCLC tissues ( $r=0.5220, \mathrm{P}<0.0001)$. ${ }^{* *} \mathrm{P}<0.01$. PSMA3-AS1, PSMA3 antisense RNA 1; miR-409-3p, microRNA-409-3p; SPIN1, spindlin 1; NSCLC, non-small cell lung carcinoma; RT-qPCR, reverse transcription-quantitative polymerase chain reaction.

activity of WT-PSMA3-AS1-treated A549 and H460 cells, whereas the luciferase activity of MUT-PSMA3-AS1 was unaffected following miR-409-3p overexpression (Fig. 3G). According to the results of the RIP assay, PSMA3-AS1 and miR-409-3p were substantially enriched in Ago2-containing beads in A549 and H460 cells (Fig. 3H). This indicated that PSMA3-AS1 and miR-409-3p co-exist in an RNA-induced silencing complex and that PSMA3-AS1 and miR-409-3p directly interact in NSCLC cells. In short, these results demonstrated that PSMA3-AS1 acted as a molecular sponge for miR-409-3p in NSCLC cells.

PSMA3-AS1 modulates SPIN1 expression in NSCLC cells by sponging miR-409-3p. Considering that PSMA3-AS1 acts as a molecular sponge, it was hypothesized that PSMA3-AS1 may regulate the expression of SPIN1, a direct target of miR-409-3p (26), in NSCLC cells via sponging miR-409-3p. The expression levels of SPIN1 mRNA and protein in PSMA3-AS1-deficient A549 and H460 cells were determined by RT-qPCR and western blotting. Reduced PSMA3-AS1 expression substantially decreased the expression of SPIN1 mRNA (Fig. 4A) and protein (Fig. 4B) in A549 and H460 cells. To perform rescue experiments, miR-409-3p inhibitor or NC inhibitor was transfected into A549 and H460 cells.
After confirming the inhibition of miR-409-3p expression by the miR-409-3p inhibitor (Fig. 4C), rescue experiments were conducted. A549 and $\mathrm{H} 460$ cells were transfected with miR-409-3p inhibitor or NC inhibitor in the presence of si-PSMA3-AS1. SPIN1 mRNA (Fig. 4D) expression was significantly downregulated by PSMA3-AS1 knockdown, while this reduction was abrogated in A549 and H460 cells by co-transfection with the miR-409-3p inhibitor. The impact of miR-409-3p inhibitor on SPIN1 mRNA expression was determined via RT-qPCR, and the results indicated that miR-409-3p inhibitor transfection resulted in a significant increase of SPIN1 mRNA expression in A549 and H460 cells (Fig. 4E). Additionally, the si-PSMA3-AS1-mediated downregulation of SPIN1 protein expression was restored in A549 and H460 cells after miR-409-3p inhibitor co-transfection (Fig. 4F). Furthermore, SPIN1 was upregulated in NSCLC tissues (Fig. 4G) and was positively associated with PSMA3-AS1 expression (Fig. 4H; r=0.5220, $\mathrm{P}<0.0001$ ). Collectively, these results clearly demonstrated that PSMA3-AS1 positively regulated SPIN1 expression in NSCLC cells by competitively binding to miR-409-3p.

PSMA3-AS1-knockdown inhibitory activities in NSCLC cells are counteracted by miR-409-3p inhibition or SPIN1 upregulation. 

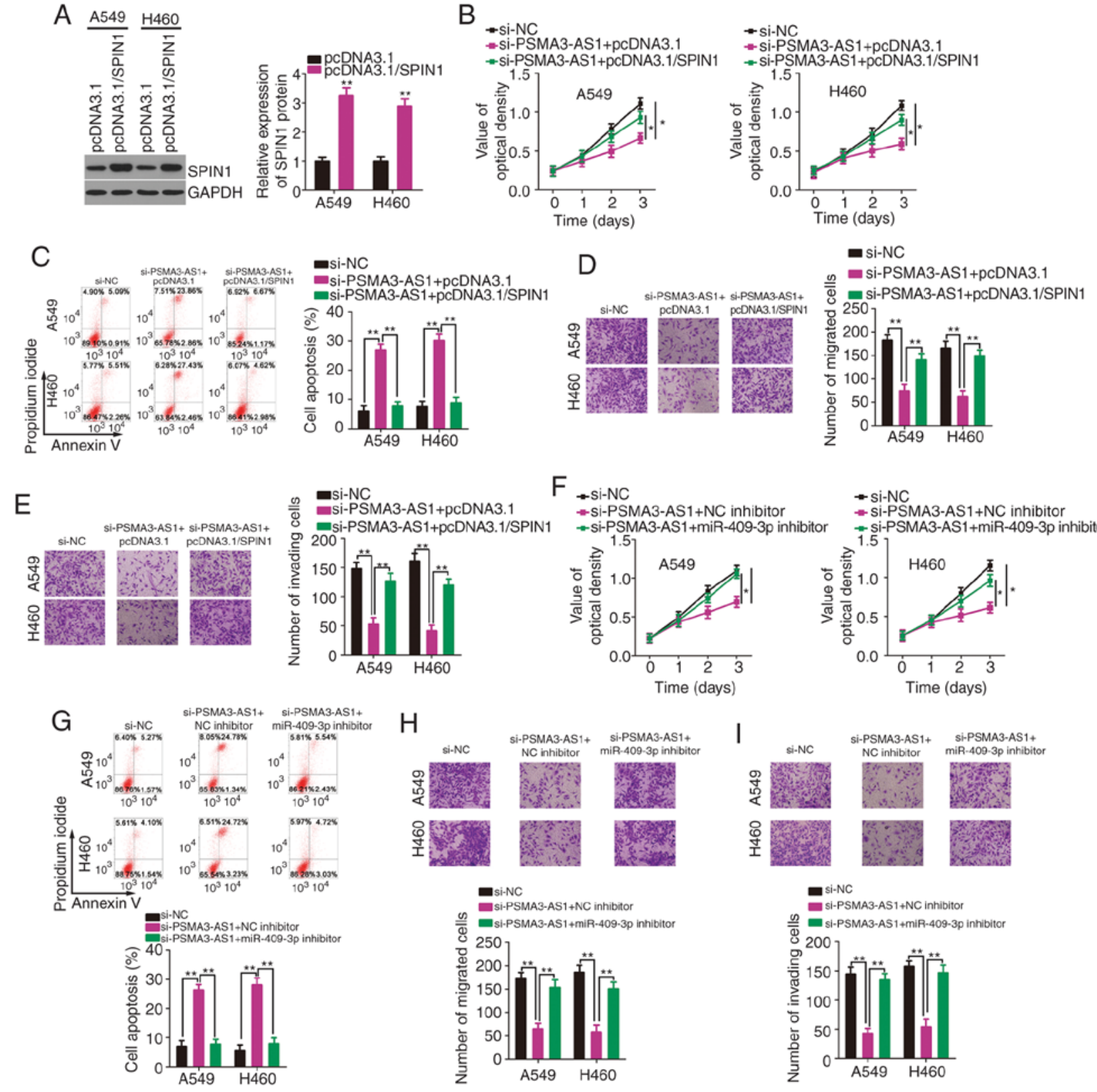

Figure 5. Overexpression of SPIN1 or inhibition of miR-409-3p counteracts PSMA3-AS1 depleted-induced inhibition of NSCLC cell proliferation, migration, and invasion, as well as the increase in cell apoptosis. (A) Western blotting was used to determine the SPIN1 protein level in A549 and H460 cells after pcDNA3.1/SPIN1 or pcDNA3.1 transfection. (B and C) A549 and H460 cells were co-transfected with pcDNA3.1/SPIN1 or pcDNA3.1 and si-PSMA3-AS1. The assessment of cell proliferation and apoptosis was performed via the CCK-8 assay and flow cytometric analysis, respectively. (D and E) The evaluation of migration and invasion in the cells described above was conducted by Transwell migration and invasion assays. (F-I) The miR-409-3p inhibitor or NC inhibitor, along with si-PSMA3-AS1, was transfected into A549 and H460 cells. The proliferation, apoptosis, migration, and invasion were determined by the CCK-8 assay, flow cytometric analysis, and Transwell migration and invasion assays, respectively. ${ }^{*} \mathrm{P}<0.05$ and ${ }^{* *} \mathrm{P}<0.01$. SPIN1, spindlin 1; miR-409-3p, microRNA-409-3p; PSMA3-AS1, PSMA3 antisense RNA 1; NSCLC, non-small cell lung carcinoma; CCK-8, Cell Counting Kit-8.

To further investigate whether the miR-409-3p/SPIN1 axis mediates the oncogenic actions of PSMA3-AS1 in NSCLC cells, rescue experiments were designed and conducted. Before these experiments, SPIN1 expression was determined to be increased in A549 and $\mathrm{H} 460$ cells following transfection with pcDNA3.1/SPIN1 (Fig. 5A). Subsequently, PSMA3-AS1-depleted A549 and H460 cells were co-transfected with pcDNA3.1/SPIN1 or an empty pcDNA3.1 plasmid. PSMA3-AS1 knockdown significantly inhibited A549 and $\mathrm{H} 460$ cell proliferation (Fig. 5B) and promoted cell apoptosis (Fig. 5C), whereas pcDNA3.1/SPIN1 co-transfection abolished these effects. In addition, restoring SPIN1 expression attenuated PSMA3-AS1 knockdown-induced suppression of A549 and H460 cell migration (Fig. 5D) and invasion (Fig. 5E). Furthermore, PSMA3-AS1 deficiency-induced inhibition of cell proliferation (Fig. 5F) and enhancement of cell apoptosis (Fig. 5G) were rescued by miR-409-3p inhibition. Similarly, the impaired migratory (Fig. 5H) and invasive (Fig. 5I) abilities following PSMA3-AS1 knockdown were restored with the co-transfection of the miR-409-3p inhibitor. Collectively, PSMA3-AS1 promoted the malignant characteristics of NSCLC cells via the miR-409-3p/SPIN1 axis.

Interference of PSMA3-AS1 impedes NSCLC tumor growth in vivo. A xenograft tumor assay was performed to reveal the 

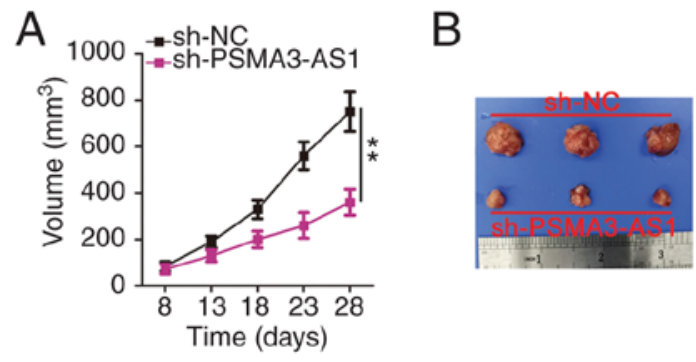

C

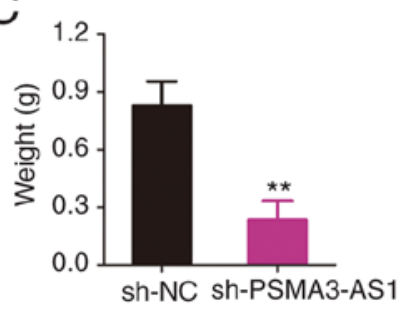

$\mathrm{D}$

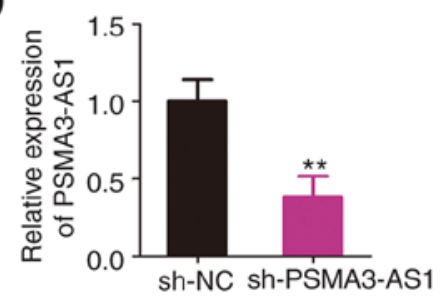

$\mathrm{E}$

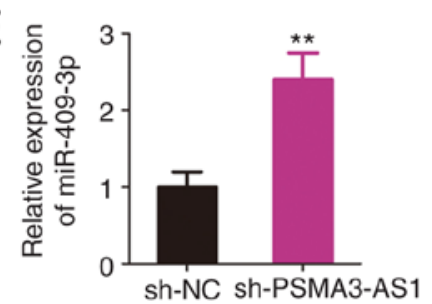

$\mathrm{F}$

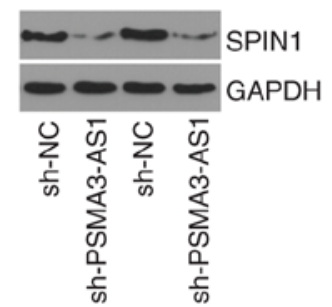

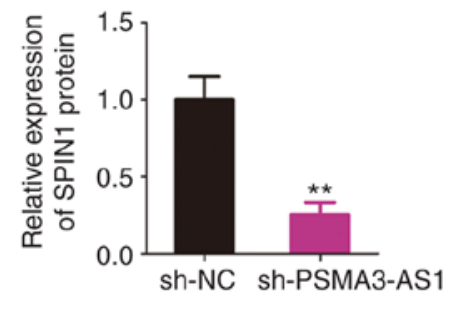

Figure 6. PSMA3-AS1 downregulation inhibits NSCLC tumor growth in vivo. (A) The tumor volume was recorded every 5 days, and the growth curves were drawn accordingly. (B) Representative images of the tumor xenografts derived from H460 cells infected with lentivirus expressing sh-PSMA3-AS1 or sh-NC. (C) The weight of tumor xenografts was measured when mice were euthanized 4 weeks post-injection. (D and E) Total RNA was isolated from tumor xenografts collected from the sh-PSMA3-AS1 and sh-NC groups and used in the detection of PSMA3-AS1 and miR-409-3p expression via RT-qPCR. (F) Protein expression of SPIN1 in xenograft mice tumor tissues was analyzed by western blotting. ${ }^{* *} \mathrm{P}<0.01$. PSMA3-AS1, PSMA3 antisense RNA 1; NSCLC, non-small cell lung carcinoma; RT-qPCR, reverse transcription-quantitative polymerase chain reaction.

role of PSMA3-AS1 in NSCLC tumor growth in vivo. H460 cells infected with lentivirus expressing sh-PSMA3-AS1 or sh-NC were subcutaneously inoculated into nude mice. The growth of tumor xenografts was reduced in the sh-PSMA3-AS1 group compared with that in the sh-NC group (Fig. 6A and B). The average weight of the tumor xenografts stably transfected with sh-PSMA3-AS1 was also decreased (Fig. 6C). Furthermore, the molecular analysis indicated that the expression of PSMA3-AS1 remained low (Fig. 6D), whereas miR-409-3p expression was increased (Fig. 6E) in the tumor xenografts collected from the sh-PSMA3-AS1 group. In addition, the tumor xenografts derived from sh-PSMA3-AS1 stably transfected $\mathrm{H} 460$ cells exhibited a significantly lower SPIN1 protein level than in the sh-NC group (Fig. 6F), as revealed by western blotting. Collectively, the downregulation of PSMA3-AS1 inhibited NSCLC tumor growth in vivo by regulating the $\mathrm{miR}-409-3 \mathrm{p} / \mathrm{SPIN} 1$ axis.

\section{Discussion}

The dysregulation of IncRNAs in various types of cancer is a growing concern among researchers $(27,28)$. Emerging studies have revealed that several lncRNAs are aberrantly expressed in NSCLC, and they have been recognized to perform oncogenic or anti-oncogenic activities during NSCLC progression $(16,29,30)$. Therefore, the identification of cancer-related lncRNAs and elucidation of the underlying mechanisms by which IncRNAs promote the aggressive phenotype of cancer cells is essential for the development of effective targets for anticancer therapies. The present study detected the expression profile of PSMA3-AS1 in NSCLC and determined its clinical relevance among patients with NSCLC. In addition, extensive experimental exploration was performed to investigate the detailed functions of PSMA3-AS1 during NSCLC progression. Furthermore, the potential mechanisms underlying the tumor-promoting actions of PSMA3-AS1 in
NSCLC cells were elucidated through a series of mechanistic experiments.

PSMA3-AS1 has been revealed to be highly expressed in esophageal squamous cell carcinoma (23). High PSMA3-AS1 expression has been demonstrated to be significantly associated with tumor size, distant metastasis, and poor prognosis in patients with esophageal squamous cell carcinoma (23). In terms of its functions in esophageal squamous cell carcinoma, exogenous PSMA3-AS1 expression has been revealed to facilitate cell growth and metastasis in vitro (23). However, there are few studies that focus on PSMA3-AS1 expression and its detailed involvement in NSCLC. The results of the present study revealed that PSMA3-AS1 was upregulated in NSCLC tissues and cell lines. Its overexpression was closely associated with TNM stage and lymph node metastasis among patients with NSCLC. Patients with NSCLC with high PSMA3-AS1 expression had shorter overall survival compared with patients with low PSMA3-AS1 expression. In addition, knockdown of PSMA3-AS1 resulted in a decrease in cell proliferation, migration, and invasion in vitro, as well as an increase in cell apoptosis. Furthermore, knockdown of PSMA3-AS1 impaired NSCLC tumor growth in vivo. Herein, it was revealed that si-PSMA3-AS1 transfection resulted in a reduction of NSCLC cell proliferation at $72 \mathrm{~h}$, and migration and invasion at $24 \mathrm{~h}$, whereas its knockdown induced apoptosis at $48 \mathrm{~h}$ post-transfection. Difference in timing observed for each effect may be attributed to the different effects of PSMA3-AS1 silencing on function-related protein expression. We aim to address this issue further in future studies.

Mechanistically, ceRNAs have been identified as an important group of post-transcriptional regulators that contribute to carcinogenesis and cancer progression by regulating their target genes through a miRNA-mediated mechanism (31). Accumulating evidence has revealed that lncRNAs localized in the cytoplasm function as molecular sponges to sequester miRNAs and consequently release 


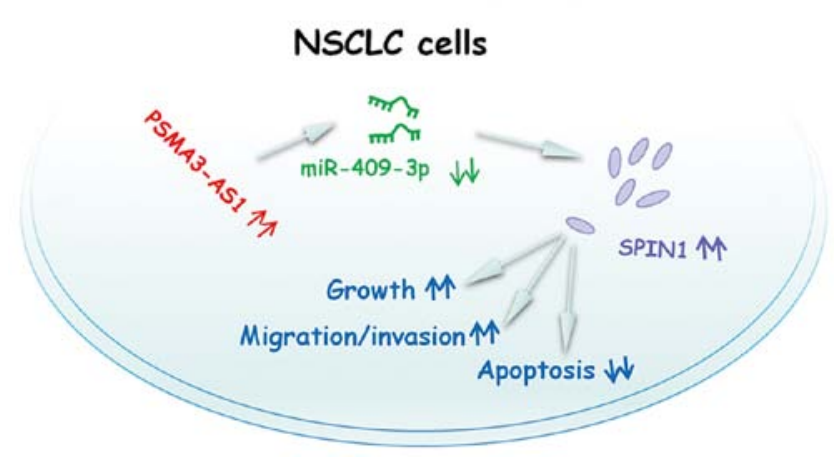

Figure 7. Schematic diagram of the PSMA3-AS1/miR-409-3p/SPIN1 pathway in NSCLC. PSMA3-AS1 aggravates the progression of NSCLC by sponging miR-409-3p and thereby increasing SPIN1 expression. PSMA3-AS1, PSMA3 antisense RNA 1; miR-409-3p, microRNA-409-3p; SPIN1, spindlin 1; NSCLC, non-small cell lung carcinoma.

their downstream target mRNAs to regulate cancer progression (25). To identify the molecular events underlying the oncogenic activities of PSMA3-AS1 in NSCLC, its subcellular localization was first evaluated. It was confirmed that PSMA3-AS1 was mainly localized in the cytoplasm of NSCLC cells, indicating that PSMA3-AS1 may function as a miRNA molecular sponge.

Next, bioinformatics analysis was performed to identify the miRNAs that potentially interact with PSMA3-AS1. miR-409-3p has been reported to perform tumor-suppressive roles in NSCLC (26). Therefore, miR-409-3p was selected for subsequent experimental verification. The RT-qPCR analysis confirmed that the downregulation of PSMA3-AS1 increased the expression of miR-409-3p in NSCLC cells. In addition, miR-409-3p was expressed at low levels in NSCLC, which was consistent with previous findings (26). The direct binding interaction between miR-409-3p and PSMA3-AS1 in NSCLC cells was verified by luciferase reporter and RIP assays. SPIN1 was identified as a downstream gene of miR-409-3p (26); therefore, it was hypothesized that PSMA3-AS1 could regulate SPIN1 expression in NSCLC cells by competitively sponging miR-409-3p, thereby driving the malignant properties of NSCLC cells. In agreement with our hypothesis, the expression level of SPIN1 was positively regulated by PSMA3-AS1 in NSCLC cells. Additional studies demonstrated that SPIN1 was highly expressed in NSCLC cells and positively correlated with PSMA3-AS1 expression. Rescue experiments further validated that the sponging of miR-409-3p was essential for the regulation of SPIN1 expression by PSMA3-AS1 in NSCLC cells. Collectively, a novel ceRNA molecular pathway involving PSMA3-AS1, miR-409-3p, and SPIN1 was identified in NSCLC cells.

miR-409-3p has been revealed to be downregulated in NSCLC and significantly related to histological stage, tumor size, pleural invasion, and metastasis (26). Notably, the overall survival and disease-free survival of patients with NSCLC with low miR-409-3p expression were shorter than that of patients with high miR-409 expression (26). SPIN1, a member of the SPIN/SSTY gene family, is known to be a direct target of miR-409-3p in NSCLC cells (26). It has emerged as an essential regulator of cancer initiation and progression $(32,33)$.
The results of the present study indicated that PSMA3-AS1 acted as a ceRNA for miR-409-3p and thereby enhanced SPIN1 expression. Rescue experiments demonstrated that decreasing miR-409-3p or increasing SPIN1 abrogated the effects of PSMA3-AS1-knockdown in NSCLC cells. Collectively, the present results revealed that PSMA3-AS1 executed its oncogenic activities in NSCLC cells by sponging miR-409-3p to increase SPIN1 expression. Therefore, the PSMA3-AS1/miR-409-3p/SPIN1 pathway (Fig. 7) may contribute to the progression of NSCLC.

In conclusion, it was demonstrated that PSMA3-AS1 functioned as an oncogenic IncRNA in NSCLC. PSMA3-AS1 sponged miR-409-3p to increase SPIN1 expression and promote the aggressive characteristics of NSCLC cells. The present study suggested that the PSMA3-AS1/miR-409-3p/SPIN1 pathway could be an attractive target for NSCLC therapies.

\section{Acknowledgments}

Not applicable.

\section{Funding}

The present study was supported by the Jilin Provincial Department of Health Research Project (grant nos. 2018J084 and 2014Q004) and the Jilin Science and Technology Bureau Medical and Health Key Projects (grant no. 201830461).

\section{Availability of data and materials}

The datasets used and/or analyzed during the present study are available from the corresponding author on reasonable request.

\section{Authors' contributions}

LWa and JP designed the study. LWa, LWu and JP performed all the experiments. LWa and JP wrote the manuscript. All authors reviewed and edited the manuscript. All authors read and approved the manuscript and agree to be accountable for all aspects of the research in ensuring that the accuracy or integrity of any part of the work are appropriately investigated and resolved.

\section{Ethics approval and consent to participate}

All participants provided written informed consent, and this study was approved by the Ethics Committee of the Affiliated Hospital of Beihua University. All experimental steps were conducted in accordance with the Declaration of Helsinki. Animal experiments were performed under the approval of the Animal Ethics Committee of the Affiliated Hospital of Beihua University.

\section{Patient consent for publication}

Not applicable.

\section{Competing interests}

The authors declare that they have no competing interests. 


\section{References}

1. Bray F, Ferlay J, Soerjomataram I, Siegel RL, Torre LA and Jemal A: Global cancer statistics 2018: GLOBOCAN estimates of incidence and mortality worldwide for 36 cancers in 185 countries. CA Cancer J Clin 68: 394-424, 2018.

2. Siegel RL, Miller KD and Jemal A: Cancer statistics, 2019. CA Cancer J Clin 69: 7-34, 2019.

3. Avelino CU, Cardoso RM, Aguiar SS and Silva MJ: Assessment of quality of life in patients with advanced non-small cell lung carcinoma treated with a combination of carboplatin and paclitaxel. J Bras Pneumol 41: 133-142, 2015.

4. Duma N, Santana-Davila R and Molina JR: Non-small cell lung cancer: Epidemiology, screening, diagnosis, and treatment. Mayo Clin Proc 94: 1623-1640, 2019.

5. Keskin S, Kutluk AC and Tas F: Prognostic and predictive role of angiogenic markers in non-small cell lung cancer. Asian Pac J Cancer Prev 20: 733-736, 2019.

6. Martin P and Leighl NB: Review of the use of pretest probability for molecular testing in non-small cell lung cancer and overview of new mutations that may affect clinical practice. Ther Adv Med Oncol 9: 405-414, 2017.

7. Ponting CP, Oliver PL and Reik W: Evolution and functions of long noncoding RNAs. Cell 136: 629-641, 2009.

8. Bhan A, Soleimani M and Mandal SS: Long noncoding RNA and cancer: A new paradigm. Cancer Res 77: 3965-3981, 2017.

9. Prensner JR and Chinnaiyan AM: The emergence of lncRNAs in cancer biology. Cancer Discov 1: 391-407, 2011.

10. Lin H, Li P,Zhang N, Cao L, Gao YF and Ping F: Long non-coding RNA MIR503HG serves as a tumor suppressor in non-small cell lung cancer mediated by wnt1. Eur Rev Med Pharmacol Sci 23 10818-10826, 2019.

11. Wang Z, Zhang J, Yang B, Li R, Jin L, Wang Z, Yu H, Liu C, Mao Y and You Q: Long intergenic noncoding RNA 00261 acts as a tumor suppressor in non-small cell lung cancer via regulating miR-105/FHL1 axis. J Cancer 10: 6414-6421, 2019.

12. Shangguan WJ, Liu HT, Que ZJ, Qian FF, Liu LS and Tian JH TOB1-AS1 suppresses non-small cell lung cancer cell migration and invasion through a ceRNA network. Exp Ther Med 18 4249-4258, 2019.

13. Yu L, Chen D and Song J: LncRNA SNHG16 promotes non-small cell lung cancer development through regulating EphA2 expression by sponging miR-520a-3p. Thorac Cancer 11: 603-611, 2020

14. Li Z and Wang Y: Long non-coding RNA FTH1P3 promotes the metastasis and aggressiveness of non-small cell lung carcinoma by inducing epithelial-mesenchymal transition. Int J Clin Exp Pathol 12: 3782-3790, 2019.

15. Kang Y, Jia Y, Wang Q, Zhao Q, Song M, Ni R and Wang J: Long noncoding RNA KCNQ1OT1 promotes the progression of non-small cell lung cancer via regulating miR-204-5p/ATG3 axis. Onco Targets Ther 12: 10787-10797, 2019.

16. Lu T, Wang Y, Chen D, Liu J and Jiao W: Potential clinical application of lncRNAs in non-small cell lung cancer. Onco Targets Ther 11: 8045-8052, 2018.

17. Wang L, Ma L, Xu F, Zhai W, Dong S, Yin L, Liu J and Yu Z: Role of long non-coding RNA in drug resistance in non-small cell lung cancer. Thorac Cancer 9: 761-768, 2018.
18. Mohr AM and Mott JL: Overview of microRNA biology. Semin Liver Dis 35: 3-11, 2015.

19. Bartel DP: MicroRNAs: Genomics, biogenesis, mechanism, and function. Cell 116: 281-297, 2004.

20. Anastasiadou E, Jacob LS and Slack FJ: Non-coding RNA networks in cancer. Nat Rev Cancer 18: 5-18, 2018.

21. Liang Y, Zhang C, Ma MH and Dai DQ: Identification and prediction of novel non-coding and coding RNA-associated competing endogenous RNA networks in colorectal cancer. World J Gastroenterol 24: 5259-5270, 2018.

22. Liz $\mathbf{J}$ and Esteller M: lncRNAs and microRNAs with a role in cancer development. Biochim Biophys Acta 1859: 169-176, 2016.

23. Qiu BQ, Lin XH, Ye XD, Huang W, Pei X, Xiong D, Long X, Zhu SQ, Lu F, Lin K, et al: Long non-coding RNA PSMA3-AS1 promotes malignant phenotypes of esophageal cancer by modulating the miR-101/EZH2 axis as a ceRNA. Aging (Albany NY) 12: 1843-1856, 2020.

24. Livak KJ and Schmittgen TD: Analysis of relative gene expression data using real-time quantitative PCR and the 2(-Delta Delta C(T)) method. Methods 25: 402-408, 2001.

25. Abdollahzadeh R, Daraei A, Mansoori Y, Sepahvand M, Amoli MM and Tavakkoly-Bazzaz J: Competing endogenous RNA (ceRNA) cross talk and language in ceRNA regulatory networks: A new look at hallmarks of breast cancer. J Cell Physiol 234: 10080-10100, 2019.

26. Song Q, Ji Q, Xiao J, Li F, Wang L, Chen Y, Xu Y and Jiao S: miR-409 inhibits human non-small-cell lung cancer progression by directly targeting SPIN1. Mol Ther Nucleic Acids 13: 154-163, 2018.

27. Abildgaard C, Do Canto LM, Steffensen KD and Rogatto SR Long non-coding RNAs involved in resistance to chemotherapy in ovarian cancer. Front Oncol 9: 1549, 2020.

28. Wu P, Mo Y, Peng M, Tang T, Zhong Y, Deng X, Xiong F, Guo C, Wu X, Li Y, et al: Emerging role of tumor-related functional peptides encoded by lncRNA and circRNA. Mol Cancer 19: 22, 2020.

29. Jiang L, Li Z and Wang R: Long noncoding RNAs in lung cancer: Regulation patterns, biologic function and diagnosis implications (Review). Int J Oncol 55: 585-596, 2019.

30. Li L, Wang Y, Song G, Zhang X, Gao S and Liu H: HOX cluster-embedded antisense long non-coding RNAs in lung cancer. Cancer Lett 450: 14-21, 2019.

31. Shuwen H, Qing Z, Yan Z and Xi Y: Competitive endogenous RNA in colorectal cancer: A systematic review. Gene 645: $157-162,2018$

32. Wang JX, Zeng Q, Chen L, Du JC, Yan XL, Yuan HF, Zhai C, Zhou JN, Jia YL, Yue W and Pei XT: SPINDLIN1 promotes cancer cell proliferation through activation of WNT/TCF-4 signaling. Mol Cancer Res 10: 326-335, 2012.

33. Drago-Ferrante R, Pentimalli F, Carlisi D, De Blasio A, Saliba C, Baldacchino S, Degaetano J, Debono J, Caruana-Dingli G, Grech G, et al: Suppressive role exerted by microRNA-29b-1-5p in triple negative breast cancer through SPIN1 regulation. Oncotarget 8: 28939-28958, 2017.

This work is licensed under a Creative Commons Attribution-NonCommercial-NoDerivatives 4.0 International (CC BY-NC-ND 4.0) License. 